\title{
ARTIGOS
}

\section{À LA NAISSANCE DU MOI}

\author{
Jean Frère ${ }^{1}$ \\ jfrere@wanadoo.fr
}

RESUMO O artigo retoma algumas etapas da elaboração do "eu”"como fundamento da psuché na Antigüidade clássica. Parte de Homero, passa por Ésquilo e Sófocles, para chegar ao momento socrático.

Palavras-chave Eu; Homero; Esquilo; Sófocles; Sócrates.

ABSTRACT The article analyses some of the moments in the elaboration of the "self", as a foundation of psyche, in ancient culture. Starting from Homer, the author goes through Aeschylus and Sophocles, to arrive at the Socratic moment.

Keywords Self; Homer; Aeschylus; Sophocles; Socrates.

Le substrat de nos pensées, de nos désirs et de nos sentiments peut se désigner, en son unité et son originalité, comme notre "moi". Cette instance psychique première est devenue fondamentale dans l'élaboration des problèmes de la connaissance et de l'action, lorsque le retour de soi sur soi est devenu capital (Augustin, Descartes). Mais, dès les premiers temps de la culture grecque, l'intérêt s'est porté sur l'affirmation du moi. 
Nous retiendrons ici quelques grandes étapes dans l'élaboration de ce fond intérieur du psychisme qu'est le moi. Poésie et philosophie y ont peu à peu contribué. Nous partirons d'Homère et d'Eschyle; puis, après le tournant sophocléen, nous viendrons à l'importance du moment socratique.

Le moi -et le toi corrélatif-, c'est le "je" et le "tu" renforcés. Dans la langue grecque le "je" et le "tu" sont intégrés dans la conjugaison du verbe lui-même. Mais, quand l'affirmation du sujet de l'action se trouve réitérée, on rencontre le "moi-je" (ego) et le "toi-tu" (su). C'est spécialement au niveau du dialogue, et dans des moments charnières de celui-ci, qu'à la fois s'exprime nettement et s'affirme la présence originale du moi (ou du toi).

Cette présence, au demeurant, évolue significativement d'Homère à Socrate. Alors que chez les personnages d'Homère, d'Eschyle et de Sophocle, le moi se révèle à travers les situations tendues du combat, de la conquête et du tourment, chez Socrate le moi s'affirme par lui-même et se reconnaît dans sa manière réfléchie et critique d'envisager les choses. Passionnel d'Homère à Sophocle, le moi se fait, avec Socrate, moi réfléchissant sur lui-même et sur le monde.

\section{Homère et Eschyle: le moi dans le combat}

Chez Homère comme chez Eschyle, c'est sur fond d'idéal guerrier et par rapport au vouloir des dieux que le moi vient à s'affirmer. Il s'exprime à travers les discours, discours opposant le "moi-je" (ego) au "toi-tu" des combattants. Le moi s'affirme comme désir de se faire reconnaître comme autre que l'autre (y compris le dieu sous la dépendance duquel le héros existe). Le moi en guerre est un moi passionné, dans lequel ce sont les émotions et les sentiments qui orientent la raison. Même si la revendication d'une décision s'ouvre sur un agir extérieur, une réelle intériorité se trouve sous-jacente à la décision et à l'acte. C'est moi, non un autre, qui suis ici présent.

Dans l'affirmation de l'ego-su, l'âme du héros se manifeste à la fois comme autre et comme une. En ce sens, c'est assurément l'intériorité de l'âme qui se trouve révélée. Quand je héros dit, non pas "je" mais "moi je", quand il dit, non pas "tu" mais "toi tu", il exprime sa singularité aussi bien face aux dieux que face aux êtres humains auxquels il s'adresse. De plus, toutes les orientations de son psychisme ne font qu'un: émotions, sentiments, raisonnements, souvenirs se trouvent réunis en cette affirmation de soi qu'est le "moi-même" (ego, parfois autos). Une unité originelle et originale de ses sentiments et idées: le moi du guerrier se découvre durant le combat, il se découvre et se manifeste comme 
un moi passionné. Toutefois, ce moi comporte un large éventail d'orientations, dont l'affectivité est une composante dynamique essentielle.

Ce moi est d'abord agressif. Dans le partage des biens, il fait un choix qui exclut entièrement l'autre. Ainsi dans l'Iliade au Chant IX, Agamemnon rappelle, tout en les déplorant, les rapines qu'il a commises lors des premiers moments du siège de Troie. Dans ce rappel, le moi d'Agamemnon s'inscrit tout naturellement. "Ces erreurs, pour moi (autos), je ne les nie pas" (v. 116). "Ce sont des Lesbiennes que j'avais choisies pour moi (autos) parce qu'elles surpassaient en beauté toutes les autres de leur sexe" (v. 129-130). Ici l'autos dans l'affirmation du moi n'a point la complète assurance contenue dans ego, mais n'en implique pas moins l'expression d'un moi personnel assumé, distinct de tout autre.

C'est dès le Chant I, dans l'affrontement implacable entre le moi des deux rois, que s'exprime en sa clarté toute la violence de l'ego. Ainsi quand Agamemnon dit à Achille: "Eh bien, fuis donc, si ton cœur a telle envie. Ce n'est pas moi (oude egoge)" qui te (se) supplie de rester ici pour me plaire" (v. 173-4). "Si Phébus Apollon m'enlève Chriséis, pour moi (ego) je la ferai mener par une nef à moi et par des hommes à moi; mais à mon tour, moi (ego) j'irai jusqu'à ta baraque et j'en emmènerai la jolie Briséis, ta part à toi, pour que tu saches combien je suis plus fort que toi (pherteros sethen)" (v. 182-6). Le moi qui s'affirme dans l'agression s'exprime ici en toute sa force dominatrice, opposant la passion avide et possessive du moi d'Agamemnon et au moi de son rival Achille, et même au dieu Apollon. Sur horizon de volontés divines et d'un idéal accepté de gloire, deux moi guerriers se manifestent en leur opposition.

Les héros guerriers ou royaux du théâtre d'Eschyle affirment leur moi dans des situations où ils sont violemment confrontés à un autre moi dominateur -à un toi dominateur. Dans la première pièce de la Trilogie, lors de la célèbre scène du tapis, Clytemnestre veut à tout prix qu'Agamemnon franchisse les étoffes splendidement brodées qu'elle vient de faire dérouler pour l'honorer. En rude guerrier appréciant moins le faste que les armes, Agamemnon énergiquement s'y oppose de tout son moi. "Je le répète, ce que tu veux, pour moi (ego), je ne le puis faire sans appréhension” (v. 930). Plus loin, lorsque la pièce s'achève, Clytemnestre et Egisthe, au lendemain du double meurtre d'Agamemnon et de Cassandre, assument leur pouvoir sur la cité sanglante. Clytemnestre dit: "Dédaigne ces vains aboiements. Maîtres de ce palais (kratounte), toi avec moi (ego kai su) nous saurons bien rétablir l'ordre" (v. 672-3). Chez Eschyle comme chez Homère, on trouve ainsi une première forme de manifestation du moi: le moi des héros guerriers ou royaux se révèle en situation, cette situation 
est le combat, et dans ce combat s'exprime leur désir ambitieux de gloire et d'avoir se profilant sur fond de dieux eux-mêmes farouches et redoutables.

Outre le moi qui se révèle agressif dans une situation de violentes divergences, il y a le moi qui se découvre et se manifeste à travers l'audace dont il fait preuve dans l'infortune. D'abord, l'audace généreuse. Voici Priam au Chant XXIV venu rencontrer Achille sous sa tente au lendemain de la mort d'Hector. Priam dit: "Respecte les dieux, Achille, et songeant à ton père, prends pitié de moi. Moi (ego) j'ai osé (etlen) ce que jamais encore n'a osé mortel ici-bas, j'ai porté à mes lèvres les mains de l'homme qui m'a tué mes enfants" (v. 503-6). Le moi audacieux généreux se rencontre aussi chez Eschyle. Tel se manifeste le moi de Prométhée. Zeus voulait anéantir la race des hommes. Prométhée réagit: “A ce projet, nul ne s'opposait que moi (plen emou). Moi (ego), j'ai eu cette audace (etolmesa), j'ai libéré les hommes" (v. 234-5). "Ils (les hommes) faisaient tout sans recourir à la raison (gnômè), jusqu'au moment où moi je (ego) leur appris la science ardue des levers et des couchers des astres" (v. 456-458). "Et moi l'infortuné, qui ai pour les mortels trouvé telles inventions, je ne possède pas moi-même (autos) aujourd'hui le secret qui me délivrerait de ma misère présente" (v. 470-1). Le moi dans l'audace généreuse affirme sa singularité, voire sa solitude face à autrui -hommes ou dieux. L'âme du héros (Priam, Prométhée) est ici radicalement elle-même, opposant sa raisonnable générosité aux conventions humaines ou au vouloir divin. Le moi est renvoyé à lui-même, en sa relation avec les autres, certes, mais pourtant à l'écart des autres.

Mais le moi audacieux se manifeste aussi, redoutable, dans le comportement cruel et meurtrier des héros d'Homère et d'Eschyle. Ainsi, les figures de Clytemnestre et d'Egisthe, dans la dernière partie de l'Agamemnon, en sont témoignage. Agamemnon est mort. Clytemnestre s'entretient avec le Coryphée: "Vous me tâtez, vous me croyez une femme irréfléchie! Et je vous le dis, moi (ego), d'un cœur qui ne tremble pas, de toi (su), louange ou blâme, c'est tout un pour moi (me)". Plus loin, Egisthe en complice exprime son contentement: "J'étais tout désigné, moi (ego) pour ourdir ce meurtre" (v. 1604). Et encore, pour se disculper de son inaction, "la ruse clairement revenait à la femme. Pour moi (ego), le vieil ennemi, j'aurais été suspect" (v. 637). Dans l'âme meurtrière, sombres desseins rusés et haine farouche se trouvent rassemblés en une profonde unité: la cruauté se confond avec la conscience et l'affirmation du moi. Dans le moi passionné et cruel, haine et ruse s'unissent, opposant l'âme du héros vainqueur à celle du héros qui n'est plus et n'a plus la possibilité de dire "moi". 
Mais le moi passionné homérico-eschyléen ne se révèle pas seulement à travers ses desseins agressifs, grandioses ou cruels. Dans une direction inverse, le moi héroïque peut se manifester en tant que moi compréhensif. L'âme en ses profondeurs intimes est ici ouverture à l'autre, non plus opposition à l'autre. Retenons la scène du Chant VI dans laquelle Hector s'entretient un instant avec Hélène avant de repartir en hâte voir les siens et reprendre le combat. Le toi d'Hélène et le moi affectueux d'Hector alternent en des propos ici remplis de profonde affection. Hector parle à Hélène: "Toi $(s u)$ fais partir celui-ci (Alexandre); qu'il se hâte de manière à me rejoindre avant que j'aie quitté la ville; pour moi (ego), je vais vois mes serviteurs et ma femme et mon fils: puis-je savoir si je leur reviendrai encore?" (v. 363-6). Le moi se révèle ici dans l'attention compréhensive aux sentiments de l'autre.

Le moi se révèle aussi comme un vecteur de paix et de sagesse. Au Chant I de l'Iliade, on assiste à un dialogue entre héros et déesse. "Achille: que viens-tu faire encore, fille de Zeus qui tient l'égide? Athénè: pour moi (ego), je suis venue du ciel pour calmer ta fureur: ne veux-tu obéir? Toi (su) contienstoi et obéis-nous" (v. 207-14). Autre scène où le moi pacifiant se manifeste en toute clarté, c'est scène où conversent avec sérénité Nestor le très sage et Agamemnon le fougueux. "Le vieux Nestor, sagement (euphroneôn) prend la parole et dit: eh bien, je dirai, moi (ego) ce qui me parait le meilleur. Nul n'aura une idée (noô) qui vaille celle que j'ai moi depuis longtemps aussi bien qu'aujourd'hui, depuis le jour même où tu sortis enlevant la jeune Briséis, moi t'en dissuadant; mais toi, tu as cédé à ton cœur superbe, tu as fait affront à un brave, il est temps de le calmer avec d'aimables dons" (IX, v. 103-110).

Le moi passionné peut s'exprimer encore, non plus face à autrui, mais dans le retour affligé de soi sur soi. Ainsi dans le regret face à un passé à jamais révolu ou à un présent difficile à supporter. Ici affleure l'amorce d'un moi réfléchi dans lequel le moi s'interroge sur la valeur et le sens de sa propre existence. On songera à ces scènes de l'Iliade dans lesquelles, avec mélancolie, Nestor évoque ce qu'il fut et n'est plus. Ainsi on lit au Chant IV ce dialogue entre Agamemnon et Nestor. “Agamemnon: que n'as-tu, vieillard, des jarrets qui puissent obéir au cœur qu'enferme ta poitrine... Nestor: Atride, moi aussi je voudrais bien être encore le même (autos hôs emè) qu'au jour où je tuai le divin Ereuthalion" (v. 313-319).

Chez Eschyle aussi le versant réflexif du moi passionné s'affirme en toute netteté, par exemple dans des passages cruciaux des tragédies évoquant l'amertume du présent. Ainsi dans les Choéphores, lorsqu'Oreste se retourne sur lui-même, après le double meurtre de Clytemnestre et d'Egisthe. "Pour moi, errant, banni de cette terre, je fuirai par le monde, vivant ou mort, ne 
laissant que ce renom de meurtrier" (v. 1043-4). Echo retentissant au "je dis" plus haut exprimé: "Oui, je le dis (phemi), j'ai tué ma mère, à bon droit: elle avait tué mon père" (v. 1027). Amertume encore que celle de Cassandre dans l'Agamemnon au moment d'être tuée par Clytemnestre. "Cassandre: Ah, quel est ce feu. Et il marche sur moi...Apollon Lykeos, pitié, pitié pour moi, pour moi (v. 1157).

Origine, substrat et unité des émotions et pensées du héros passionné ou guerrier ou royal, le moi homérico-eshyléen s'affirme en certains moments essentiels de la vie du héros. Latent sous sa forme implicite d'un "je" et d'un "tu", il n'est alors qu'ébauche. La revendication du "moi" s'exprime en des moments cruciaux grâce à la réitération langagière de l'ego et du su. L'intériorité ainsi exprimée reste néanmoins "relative", ${ }^{2}$ toujours liée à une réalité extérieure à l'individu, car elle s'inscrit sur horizon d'un idéal déjà donné de gloire guerrière et de vouloirs transcendants, et se manifeste, non dans le silence d'une pensée solitaire, mais dans l'affrontement d'un dialogue plus souvent agressif et hautain que compréhensif.

\section{Sophocle: le moi dans la cité}

Dans le théâtre de Sophocle, on retrouve comme chez Homère et chez Eschyle, les situations de rivalité où le moi se révèle à travers son agressivité et son audace passionnées. Mais ce qui apparaît pour la première fois avec force chez Sophocle, c'est le moi tel qu'il se découvre en se confrontant aux problèmes liés à la cité. Les pièces comme Edipe Roi et Antigone s'avèrent, sur ce point, essentielles. Ici encore l'affirmation du moi en tant que confronté à un toi est l'expression la plus haute de la singularité intérieure du moi en sa différence radicale qui l'oppose à l'autre. La passion du héros se manifeste lorsqu'elle clame avec force son originalité, ici audacieuse ou agressive, là révoltée ou dominatrice, ailleurs inquiète ou abattue.

Devant le désarroi et les silences du devin Tirésias, voici Edipe clamant la belle audace qui le guida vers une Thèbes éprouvée par la présence de la Sphynx ravageuse. Audacieux, le moi d'Edipe s'affirme en tels propos: "J'arrive, moi, Edipe, ignorant de tout, et je lui ferme la bouche, sans rien connaître des présages, par ma seule présence d'esprit (gnômè)" (Edipe Roi, v. 397-8). Belle audace du moi d'CEdipe pour lequel l'ambition raisonnée et la passion généreuse de venir au secours des malheureux s'harmonisent 
sans peine. Audace séduisante, à ce point séduisante que, face à ce jeune prince ambitieux et fougueux, la grande reine Jocaste ne soupçonne à aucun moment qu'CEdipe puisse être son fils! Quant au jeune audacieux, son but est désormais de régner; le mariage rend possible ce règne, qu'accompagnera une quadruple descendance -qu'Edipe soit amoureux empressé ou qu'il ne le soit pas. Audacieux et calculateur, Edipe affirme son moi en toute sa plénitude de désir de grandeur.

Affirmé, vécu, agissant à partir de l'ardeur du désir, le moi s'affirme dans la montée d'une ambition sans mesure. Dans un dialogue serré, haineux, Créon et CEdipe s'affrontent pour revendiquer le pouvoir légitime de régner. Créon: "Moi, je te demande à toi: réponds à ton tour, ainsi que je l'ai fait pour toi" (v. 514-5). "N'ai-je pas, moi, part égale de votre pouvoir à tous deux (Jocaste et (Edipe)?" (v. 581). "Pour moi, je ne suis pas né moi-même avec le désir d'être roi, mais bien avec celui de vivre comme un roi" (v. 587-8). Le moi haineux de Créon affronte celui d'Oedipe. À qui revient le pouvoir légitime? Créon se sent exclu et son moi réagit. Edipe s'éprouve à l'aise en son pouvoir conquis. Le moi vainqueur se manifeste par son opposition violente à l'autre moi, celui de l'exclu. Les moi s'expriment et s'affirment ici sur un horizon accepté et donné, celui du pouvoir et de la gloire.

Mais Sophocle connaît aussi des formes nouvelles de la manifestation du moi. C'est le moi prenant conscience de sa dimension singulière à l'intérieur de la cité. Ainsi en est-il du moi d'Antigone face au moi de Créon. Le moi chez Créon s'affirme et se manifeste à travers le désir du pouvoir et de l'ordre dans la cité. “Aujourd'hui qu'ils ont disparu (EEdipe, Étéocle, Polynice), c'est moi qui, désormais, possède leur trône et tout leur pouvoir" (Antigone, v. 73). Moi impitoyable, n'acceptant aucune forme de résistance venant des citoyens. "Moi et Zeus m'en est témoin, Zeus qui voit tout et à toute heure -moi, je ne puis me taire quand, au lieu du salut, j'entrevois le malheur qui marche vers ma ville" (v. 184-5). Pour le roi au moi dominateur des décrets implacables s'imposent sans appel. Polynice considéré comme traître restera sans sépulture". Or la sanction punitive estimée juste par Créon trouve son fondement dans la justice redoutable de Zeus à l'égard des méchants. Le moi du tyran, avec son besoin d 'ordre dans la cité, impose au peuple sa loi, qu'il pense inspirée de Zeus.

À ce moi qui s'arrête à l'ordre apparent des cités s'oppose Antigone. Le moi d'Antigone a ceci de particulier qu'il se révèle dans une révolte qui le relie à plus haut que soi-même, à des lois plus amples que les étroites lois apparemment utiles à l'existence harmonieuse de la cité. Lois justes émanant de Zeus et des dieux infernaux, loi du respect dû à tout guerrier qui meurt les 
armes à la main. Polynice a droit à une sépulture; le geste d'Antigone a pour fondement les lois non écrites, lois de toujours. Le moi révolté crie: "Ces loislà, pouvais-je, moi, par crainte de qui que ce fût, m'exposer à leur vengeance chez les dieux?" (v. 458-459). Qu'importe, dès lors, être condamnée à mort: un moi révolté ne peut qu'assumer sans crainte son acte. "Mourir avant l'heure, je le dis, moi, bien haut, c'est tout profit, lorsqu' on vit, comme moi, au milieu des malheurs sans nombre" (v. 462-3). Et le moi révolté implique mépris d'un toi qui ne s'appuie que sur des conventions d'apparence justes. "À toi, je parais sans doute agir comme une folle" (v. 469).

Une autre face du moi que l'on trouve envisagée par Sophocle, c'est celle du retour tourmenté de soi sur soi. Moi qui s'inquiète de lui-même. Moi effrayé et abattu à propos de lui-même. Le moi de l'inquiétude sur soi se manifeste, dans le personnage d'CEdipe, en diverses étapes de son existence. Ayant appris quel destin infortuné l'attendait, il s'enfuit de Corinthe: 'Et moi, après avoir entendu, je laisse Corinthe et son territoire" (Edipe Roi, v. 7945). L'inquiétude sur soi reprend lorsque, à la suite de bien des recoupements, Edipe s'entretient avec la reine Jocaste, désormais son épouse. L'inquiétude du moi se fait ici égarement. Edipe: “Ah, comme à t'entendre, femme, je sens soudain mon âme qui s'égare (psuchès planema), ma raison qui chancelle". Jocaste: "Quelle inquiétude (merimne) te fait soudainement regarder en arrière (hypostrapheis)?” (v. 726-7).

Autre forme du retour tourmenté sur soi-même: outre l'inquiétude sur soi, c'est l'effroi qui s'empare du moi; ainsi lorsque le crime se trouve dévoilé. Si Edipe ne s'était point crevé les yeux, comment aurait-il pu pénétrer aux enfers? 'Et moi, de quels yeux, descendu aux enfers, eussé-je pu, si j’y voyais, regarder mon père et ma mère, alors que $j$ 'ai, sur tous les deux, commis des forfaits atroces" (v. 1371-13).

Pour Sophocle, ce n'est pas seulement dans des situations de tension et de combat que le moi se révèle et s'affirme. À ses yeux, le moi s'affirme en se reliant à des valeurs qui le transcendent, il s'approfondit en s'interrogeant sur la valeur de l'ordre qu'un chef impose et des desseins des dieux dans le cadre de la cité. Sophocle a encore retenu la dimension réflexive du moi, moi qui fait retour sur lui-même dans les moments d'inquiétude et d'effroi face à la révélation tardive de crimes commis dans l'ignorance. Un moi passionné, tour à tour, audacieux, révolté, inquiet sur lui-même: tel est le moi qui se manifeste -discours et action- dans les œuvres sophocléennes, sous la loi tantôt implacable tantôt clémente de Zeus. Loin d'être la marionnette des dieux, le moi humain fait voir son originalité dans ses choix personnels sur horizon de loi divine obscure. 


\section{Socrate: l’avènement du moi réfléchi}

Dans les simples énoncés des formules à la première personne, le "je" socratique reste à l'état de simple ébauche: "Je ne sais pas (ouk oida)"; "je crois" (pisteuô); je dis (legô). En revanche, dans les moments où l'ego se trouve joint au verbe exprimé à la première personne, le moi surgit en toute sa netteté et vigueur: "Pour moi (ego), en les écoutant, j'ai failli oublier qui je suis"; "pour moi, je conviendrais alors que je suis orateur" (Apologie de Socrate, 17a-c). C'est dans l'affrontement avec l'autre, (accusateur ancien, tel Aristophane, accusateurs actuels, tel Mélétos et Anytos), que le moi se révèle en sa profonde différence par rapport à autrui, mais ici avec son exigence de rationalité, de vérité, de justice, ainsi que d'exactitude historique. Le moi socratique n'est donc pas impulsif et passionné, mais réfléchi. Il s'affirme et évolue à travers une réflexion permanente.

Il s'agit d'un moi critico-rationnel sur le fond d'une vérité et d'une justice transcendantes. Ébauche de ce qui va être le moi chez les grands penseurs grecs, Platon, Aristote, Épicure, les Stoïciens: le moi face au Bien, au Premier Moteur etc. C'est, chez Socrate, à la fois un moi de l'intériorité et un moi de la réflexivité. D'une intériorité mieux cernée que chez les héros des auteurs tragiques, un moi de la réflexivité qui ne cesse de s'interroger sur ce qu'est l'âme. Intériorité et réflexivité néanmoins relatives, car elles ne sont pas ce retour radical sur soi que l'on voit s'approfondissant à partir d'Augustin, chez Montaigne, Descartes, Kant, Nietzsche, Freud, Bergson, Sartre...Ce qui devient essentiel, c'est la disposition interne de l'âme, non plus la réussite extérieure. Le juste ne s'intéresse plus au monde du pouvoir. L'hégémonie de la raison et de l'énergie du coeur, par opposition à celle de l'action glorieuse, est une exaltation de l'intérieur aux dépens de l'extérieur. Le moi de Socrate, c'est l'unité de l'ensemble des pensées et des sentiments envisagés sous la houlette de la raison. Une intériorité réfléchie laquelle demeure soumise à l'ordonnance rationnelle et belle du cosmos.

C'est dans le dialogue avec l'autre que le moi réfléchi socratique s'affirme, profilé toutefois sur horizon de réalités qui le transcendent d'une façon ou d'une autre: Zeus, Apollon, ordre du monde, voire lois justement établies d'une cité droitement menée. Dans l'Apologie, en 25c, c'est Zeus, conçu comme dieu souverainement bon, qui se trouve évoqué dans l'appréhension du moi. "Dismoi, au nom de Zeus, Mélétos, s'il vaut mieux vivre avec d'honnêtes gens ou avec des malfaiteurs". Un autre nom de dieu conçu comme parfaitement bon et véridique se rencontre en tant que fondement essentiel dans l'appréhension par Socrate de son moi d'homme sage et savant. En 21a-b, c'est Apollon qui, selon Chéréphon, dit que Socrate est le plus savant des hommes. "Pour moi, dit 
Socrate, lorsque je connus cet oracle, je me dis à moi-même (enethumoumen): que dit et que cache le dieu? Pour moi, j'ai conscience (sunoida en autô) que je ne suis savant ni peu ni beaucoup. Or il ne peut pas parler contre la vérité". Le questionnement sur le sens de la parole divine est celui d'un moi dont l'intériorité est conscience de soi qui réfléchit, qui s'interroge sur sa propre valeur, et qui engage, dans cette réflexion, le logos et le thumos.

En d'autres lieux du dialogue, on retrouve exprimée cette remontée à un fondement divin dans l'affirmation du moi qui réfléchit sur sa propre valeur. Ainsi en 33c, "Pour quelles raisons certains auditeurs prennent-ils plaisir à passer beaucoup de temps avec moi? C'est qu'il leur plaît de voir examiner ceux qui se croient savants et qui ne le sont pas. Pour moi, je l'affirme, c'est un devoir que la divinité m'a prescrit".

Être au service d'une divinité excellente se concrétise d'abord dans la nécessité d'être au service des lois justes d'une cité juste. Ce qu'exigent les lois justes et les chefs justes constitue un premier horizon du surgissement de l'ego réfléchi de Socrate. Rester ferme dans la guerre, au poste que les chefs de la cité vous ont imposé: le moi se manifeste ici en assumant courageusement sa responsabilité face à ses concitoyens. "Quiconque occupe un poste où il est placé par un chef a pour devoir, selon moi, d'y demeurer ferme quel qu'en soit le risque" (28d-e).

Néanmoins, par delà l'amour envers les concitoyens, le moi socratique s'affirme en défendant une hiérarchisation nouvelle des valeurs bousculant les idéaux traditionnels. Ce n'est ni à l'argent ni aux honneurs que le moi lucide doit se consacrer. C'est à rendre sa propre personne meilleure. Comment? Grâce au travail de l'intelligence critique (phronesis), fondement de l'amélioration du moi. Désormais le moi se penche sur le moi tout en interpellant le moi des autres. "Athéniens, quant à moi, je vous aime, mais j'obéirai aux dieux plutôt qu'à vous. Tant que j'aurai un souffle de vie, je ne cesserai pas de vous exhorter. Quoi? Tu es Athénien, et tu ne rougis pas de donner tes soins à ta fortune ainsi qu'à ta réputation et à tes honneurs; mais quant à ta raison, quant à la vérité, quant à ton âme qu'il s'agirait d'améliorer sans cesse, tu n'y songes pas" (29d). Réfléchi, le moi socratique incite ses concitoyens à la réflexion. Interrogatif sur lui-même, il est un aiguillon qui cherche à éveiller l'esprit critique d'autrui.

C'est cette incitation à la réflexion, et à la quête du bien qui s'y trouve reliée, qui fonde l'authenticité du moi de Socrate et de ceux qui engagent le dialogue avec lui. Ainsi, est-ce en se pliant à ces règles de l'examen de soi que le disciple à son tour peut reconnaître qu'il est un moi lucide, un moi raisonnable, épris de vérité, par opposition aux individus si nombreux dont le 
moi n'a point part à la justice et à la vérité. Une scène de l'Alcibiade est tout à fait éclairante. "Socrate: Lequel est-ce de nous deux qui dit que deux est plus fort qu'un d'une unité? Alcibiade: C'est moi. Socrate: Or moi j'interrogeais et toi tu répondais. Alcibiade: c'est bien cela. Socrate: Ainsi est-ce moi qui dit les choses quand j'interroge ou toi quand tu réponds? Alcibiade: C'est moi" (112e-113a). On assiste ici à la naissance d'un moi authentique chez le disciple éclairé. Le moi éclairé d'Alcibiade s'est désormais accordé au moi de Socrate pour dire que, lorsqu'il croyait savoir ce qu'est la justice, il l'ignorait. Le moi authentique naît donc du dialogue à la recherche du vrai aussi bien chez le disciple que chez Socrate. "Quand nous nous entretenons toi et moi en échangeant des propos, c'est l'âme qui parle à l'âme (he psuchè pros ten psuchen)" (Alcibiade, 130d). Le moi authentique se manifeste non tant par la parole que par la parole en quête de vérité.

Parmi les thèmes essentiels et "difficiles" (la justice, la piété, le courage...) dont débattent les “moi” sages, l'une des questions qui importe tout spécialement pour la compréhension de ce qu'est le moi est celle de l'immortalité de l'âme. Que devient le moi lorsque la mort vient anéantir notre corps? La prudence ici comme ailleurs s'impose. Savoir ce que peut être le domaine de l'au-delà ne demeure l'objet que de belles croyances. Le moi lucide est, ici encore, en quête de preuves. Mais comment dépasser ce que constatent la raison et les sens? Le corps est là sans vie, mais que devient l'âme, son devenir multiple, son unité profonde à nulle autre pareille. Deux possibilités sont à envisager. Première possibilité: si le sentiment n'existe plus, si la mort est un sommeil où l'on ne voit plus rien, "quel avantage ce doit être que de mourir! Toute la suite des temps apparaît comme une nuit unique" (Alcibiade, $40 \mathrm{~d}-\mathrm{e}$ ). Deuxième possibilité: s'il est vrai que là-bas sont réunis ceux qui sont morts, que peut-on imaginer de meilleur? Si, en arrivant chez Hadès, on trouve des juges véritables, si l'on y fait société avec Orphée, Hésiode, Homère, les héros d'Homère tels Agamemnon, Ulysse, Ajax ou Palamède, que ne donnerionsnous pas pour en jouir? Le moi du sage, alors, subsiste, retrouvant d'autres moi qui lui sont particulièrement chers, sinon dans leur quête de la vérité, du moins dans leur exigence de justice. La conception socratique de ce qu'est la mort est ainsi infiniment plus satisfaisante que les pseudo-preuves envisagées par Platon. Ici, le moi sage reste dans l'expectative d'un “ou bien ou bien”. Là où la preuve manque, le moi sage n'affirme ni ne nie.

Dans la prise de position du héros ou du penseur à l'égard des dieux et à l'égard des grands idéaux qui s'imposent à lui (la gloire, l'ordre dans la cité, la vérité) et dans son face à face avec autrui, l'homme grec, par des approches diverses, découvrit ce qu'est le moi. 
C'est d'abord dans le dialogue du héros passionné homérique ou eschyléen avec d'autres héros que l'on trouve une première manifestation de l'affirmation du moi, le moi qui se pose en s'opposant librement à l'autre, le moi pugnace. Chez Sophocle, une nouvelle originalité dans la conception du moi se dessine; elle repose sur la contestation qu'envisage le moi face au sens ambigu des paroles de Zeus concernant l'ordre dans la cité (Antigone et Créon) ou, dans l'interrogation du coupable sur le déroulement de sa propre vie (Edipe): c'est le moi inquiet.

Quand on passe de la poésie à la philosophie, une tout autre conception vient à naître. Chez Socrate, c'est la conscience réfléchie du moi sur luimême qui, unissant thumos et logos, s'interroge sur les valeurs et se libère des croyances du grand nombre: pouvoir, honneurs, argent passent au second plan devant la quête du juste et du vrai. Dans le dialogue entre le moi du maître et le moi du disciple, l'accord se fait sur le savoir et le non savoir; et ce moi authentique, réfléchi et sage s'oppose radicalement au moi désordonné et (ou) injuste qu'est celui des foules, esclaves de leur incapacité de réfléchir droitement. C'est, interrogeant autrui et s'interrogeant soi-même, le moi questionnant.

Le moi grec est fondamentalement un moi situationnel. C'est la sollicitation d'une situation extérieure qui porte l'individu à se découvrir agressif, audacieux, inquiet, tourmenté, déchiré entre des tendances contraires ou désireux de faire la paix. C'est encore une situation extérieure qui porte l'individu à découvrir en lui un moi qui se structure par rapport à des valeurs, à des dieux invisibles. Mais, d'Homère à Socrate, nous assistons à une intériorisation. Au moi entièrement extraverti des personnages homériques et eschyléens, succède le moi intraverti de l'Antigone de Sophocle, qui exprime à Créon cela même qui, en structurant son individualité, fonde sa révolte. Quant au moi socratique, tout en restant relié aux événements extérieurs, il s'affirme fondamentalement par rapport à des principes transcendants. Le moi grec s'intériorise progressivement sans jamais se détourner du monde extérieur. Au contraire, c'est le monde extérieur qui invite le moi grec à s'interroger sur lui-même et à aborder les autres à partir de cette interrogation. 